04,09

\title{
Роль дырочных ловушек в термолюминесценции дозиметрического пика в анион-дефектных монокристаллах $\alpha-\mathrm{Al}_{2} \mathrm{O}_{3}$
}

\author{
(C) С.В. Никифоров, В.С. Кортов \\ Уральский федеральный университет им. Б.Н. Ельцина, \\ Екатеринбург, Россия \\ E-mail: s.v.nikiforov@urfu.ru
}

(Поступила в Редакцию 27 февраля 2017 г.)

\begin{abstract}
Исследованы термолюминесцентные свойства анион-дефектных монокристаллов оксида алюминия с различной полушириной основного (дозиметрического) пика при $400-500 \mathrm{~K}$. Получены новые экспериментальные подтверждения дырочной природы ловушек, ответственных за высокотемпературную часть данного пика. Введение в рассмотрение дырочных центров захвата позволило теоретически обосновать экспериментально наблюдаемые зависимости интенсивности термолюминесценции (ТЛ), температурного положения и полуширины основного пика от степени заполнения глубоких ловушек. Дырочная природа ловушек высокотемпературной части основного ТЛ-пика подтверждается при анализе особенностей ТЛ мелких центров захвата, обусловливающих ТЛ при $350 \mathrm{~K}$, а также при изучении температурных изменений спектра ТЛ основного пика.
\end{abstract}

DOI: $10.21883 /$ FTT.2017.09.44839.051

\section{1. Введение}

Интерес к изучению термолюминесценции (ТЛ) анион-дефектных монокристаллов оксида алюминия $\left(\alpha-\mathrm{Al}_{2} \mathrm{O}_{3}\right)$ обусловлен их широким практическим применением в качестве люминесцентных детекторов ионизирующих излучений $[1,2]$. Источником дозиметрической информации в них является интенсивность или светосумма ТЛ-пика при 400-500 K (скорость нагрева $2 \mathrm{~K} / \mathrm{s}$ ), который, как и связанные с ним ловушки, в литературе получил название дозиметрического (основного) пика. Высокий выход ТЛ обеспечивается выращиванием кристаллов оксида алюминия в сильно восстановительных условиях, которые приводят к формированию анионных дефектов (кислородных вакансий) в высоких концентрациях. Кислородные вакансии, захватившие два и один электрон, образуют $F$ - и $F^{+}$-центры соответственно [3], которые являются основными люминесцирующими центрами в анион-дефектном $\alpha-\mathrm{Al}_{2} \mathrm{O}_{3}$.

Известно, что форма и температурное положение дозиметрического пика ТЛ в анион-дефектных кристаллах оксида алюминия варьируются от образца к образцу. Можно выделить образцы данных кристаллов с узкими (полуширина пика менее $40 \mathrm{~K}$ при скорости нагрева $2 \mathrm{~K} / \mathrm{s}$ ) и широкими пиками, полуширина которых может достигать величины более $50 \mathrm{~K}[4,5]$. При этом уширение пика происходит в сторону более высоких температур, что вызывает смещение в высокотемпературную область положения его максимума. В ряде образцов кривая ТЛ имеет ярко выраженную двухпиковую структуру [5-7].

Указанные вариации параметров основного ТЛ-пика свидетельствуют о наличии в энергетическом спектре ловушек, ответственных за данный пик, нескольких составляющих. Попытки объяснить ТЛ основного пика в анион-дефектном $\alpha-\mathrm{Al}_{2} \mathrm{O}_{3}$ как суперпозицию вклада нескольких центров захвата предпринимались в ряде работ [8-11]. Есть мнение, что основной пик может быть обусловлен равномерным распределением ловушек по энергии, ширина которого коррелирует с полушириной пика [4]. Аппроксимация широкого ТЛ-пика уравнением общего порядка кинетики, учитывающим две ловушки, была проведена другими авторами [6]. В работе [7] было показано, что в образцах с узкими и уширенными ТЛ-пиками наблюдаются различия в дозовых зависимостях параметров ТЛ. При этом широкий ТЛ-пик может быть обусловлен суперпозицией двух ловушек с максимумами ТЛ при 190 и $250^{\circ} \mathrm{C}$. Выводы о сложной многокомпонентной природе основных ловушек подтверждаются также результатами исследования оптически стимулированной люминесценции [12-14]. Так, в работе [12] были обнаружены различия в оптическом обесцвечивании низко- и высокотемпературных составляющих ТЛ-пика при 400-500 К. При исследовании кривых линейно-модулированной оптически стимулированной люминесценции в различных образцах анион-дефектных монокристаллов оксида алюминия установлено, что основная ловушка включает в себя не менее трех элементарных компонент [12,14].

Важной проблемой, решение которой необходимо для понимания механизмов ТЛ основного пика в исследуемых кристаллах, является установление электронной или дырочной природы ловушек, ответственных за данный пик. В работе [5] на основании исследования изменений концентрации $F^{+}$-центров в температурном диапазоне дозиметрического пика (400-500K) было установлено, что низкотемпературная часть ловушек имеет электронную природу. Данную точку зрения можно считать общепризнанной, так как она поддерживается другими авторами $[7,10,13]$. Менее изучена 
природа высокотемпературных ловушек, ответственных за дозиметрический ТЛ-пик в анион-дефектном $\alpha-\mathrm{Al}_{2} \mathrm{O}_{3}$. Предположение о возможной дырочной природе данных центров было высказано в работе [8] на основе обнаруженных различий спектрального состава ТЛ низкои высокотемпературной части пика при 400-500 K в образцах корунда, легированного магнием [15]. Это предположение использовалось для объяснения различий в форме кривых фототрансферной люминесценции, стимулированной одновременно оптическим и термическим воздействием, в образцах с узким и двойным ТЛ-пиком [7].

Другим возможным способом установления электронной или дырочной природы центров захвата является исследование эффектов влияния состояния заселенности глубоких ловушек на ТЛ основного пика $[10,16,17]$. Известно, что электронные и дырочные глубокие ловушки обнаруживаются в исследуемых кристаллах по данным измерения высокотемпературной ТЛ [17-20] и другим косвенным признакам (фототрансферная ТЛ, изменение выхода ТЛ и повышение светочувствительности материала) $[5,8,10,13,16,17,21]$. Их влияние на ТЛ основного пика обусловлено конкурирующими процессами в захвате носителей заряда, которые могут быть описаны в рамках известных кинетических моделей [22-24]. При этом различия в механизмах конкурирующего взаимодействия низко- и высокотемпературных основных ловушек с электронными и дырочными глубокими центрами могут приводить к изменению параметров ТЛ-пика при 400-500 K [10]. В работе [11] нами было установлено изменение вида зависимости интенсивности ТЛ основного пика от степени заполнения глубоких центров в образцах с широкими ТЛ-пиками по сравнению с наблюдаемым для образцов с узкими ТЛ-пиками. Полученные результаты позволили предположить дырочную природу высокотемпературной части основных ловушек, что согласуется с данными работ [7,8]. Для теоретического описания наблюдаемых эффектов изменения выхода ТЛ была предложена кинетическая модель, учитывающая процессы переноса зарядов с участием электронных и дырочных основных и глубоких ловушек [11].

Следует отметить, что выводы о дырочной природе ловушек, ответственных за уширение основного пика, сделанные в работе [11], были основаны на анализе только лишь одного эффекта (изменения выхода ТЛ). Поэтому для обоснования дырочной природы высокотемпературной части основных ловушек требуется проведение дополнительных исследований влияния глубоких ловушек на другие параметры ТЛ-пика при 400-500 К (температурное положение и форму) с одновременным уточнением механизмов процессов переноса зарядов с участием основных и глубоких ловушек в рамках кинетических представлений. Изменение кривых ТЛ образцов $\alpha-\mathrm{Al}_{3} \mathrm{O}_{3}$ с различной заселенностью глубоких центров изучалось ранее в работе [10]. При этом заполнение глубоких ловушек варьировалось путем изменения дозы УФ- и бета-излучения, а также температуры отжига кристаллов. Однако детальный анализ раздельного влияния глубоких ловушек различной энергетической глубины на параметры кривой ТЛ с разной полушириной основного пика в работе [10] не проводился.

Необходимо отметить также, что в модели, предложенной в работе [11], предполагалась дырочная природа очень глубоких ловушек (при $T>1050 \mathrm{~K}$ ), что не является строго установленным фактом. Относительно природы этих ловушек есть различные точки зрения. В работах $[10,16]$ они рассматриваются как электронные, а в работах $[17,21]$ - как дырочные. В связи с этим для повышения достоверности установления дырочной природы ловушек высокотемпературной части основного ТЛ-пика при 400-500 K необходимо привлечение дополнительных данных об особенностях процессов переноса заряда с участием не только глубоких, но и других (в частности, мелких) ловушек, ответственных за ТЛ-пик при $350 \mathrm{~K}$ в исследуемых кристаллах $[25,26]$. Представляется также важным подтверждение дырочной природы дозиметрических ловушек на основе результатов исследования спектрального состава ТЛ-пика при 400-500 K, которые напрямую не связаны с конкурирующими процессами переноса заряда.

Настоящая работа посвящена экспериментальному исследованию и компьютерному моделированию особенностей ТЛ в образцах анион-дефектных монокристаллов оксида алюминия, характеризующихся различной полушириной основного ТЛ-пика, с целью обоснования природы центров захвата, ответственных за данный пик.

\section{2. Образцы и экспериментальные методы}

Исследовались образцы стандартных ТЛ-детекторов ТЛД-500К, изготовленных из анион-дефектных монокристаллов $\alpha-\mathrm{Al}_{2} \mathrm{O}_{3}$, выращенных методом Степанова в восстановительных условиях [27]. Образцы имели форму дисков толщиной $1 \mathrm{~mm}$, диаметром $5 \mathrm{~mm}$ и были полупрозрачными. Для возбуждения ТЛ основного пика образцы облучались при комнатной температуре бета-излучением изотопа $\mathrm{Sr}^{90} / \mathrm{Y}^{90}$. Для заполнения глубоких ловушек использовалось нефильтрованное УФ-излучение ртутной лампы ДРТ-240. ТЛ регистрировалась с помощью ФЭУ-130 с максимумом спектральной чувствительности при 400-420 nm. Скорость нагрева образцов составляла $2 \mathrm{~K} / \mathrm{s}$. Оборудование для измерения ТЛ со спектральным разрешением подробно описано в работе [28].

\section{3. Результаты и их обсуждение}

Для исследований была отобрана партия образцов анион-дефектного оксида алюминия с разбросом 


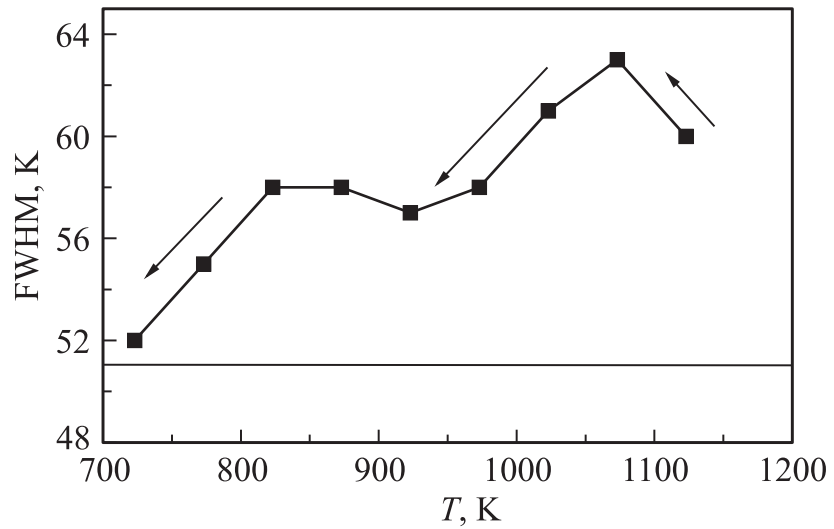

Рис. 1. Зависимость полуширины (FWHM) ТЛ-пика при $400-500 \mathrm{~K}$ от температуры облучения УФ-излучением для образцов с широким основным пиком. Горизонтальная линия соответствует исходному значению полуширины.

ТЛ-чувствительности к излучению не более $5 \%$. Чувствительность оценивалась по интенсивности ТЛ в основном пике при $400-500 \mathrm{~K}$ после облучения образцов малой (тестовой) дозой от бета-источника $(8 \mathrm{mGy})$ на линейном участке дозовой характеристики. В этих условиях изменение состояния заселенности глубоких ловушек можно считать пренебрежимо малым. Исследовались образцы с узкими ТЛ-пиками (полушириной менее $40 \mathrm{~K}$ ) и с широкими пиками (полушириной более $50 \mathrm{~K})$.

Для установления новых закономерностей влияния глубоких ловушек на ТЛ образцов с различной формой кривой термовысвечивания исследовалось влияние температуры заполнения глубоких ловушек на положение максимума и полуширину дозиметрического пика ТЛ. Нами использовалась методика эксперимента, предложенная в работе [11], где изучалось влияние глубоких центров захвата на ТЛ-чувствительность. Ловушки заполнялись УФ-излучением последовательно, начиная с самых глубоких, при уменьшении температуры возбуждения в диапазоне $1123-723 \mathrm{~K}$ с шагом $50 \mathrm{~K}$. ТЛ основного пика регистрировалась в полосе свечения $F$-центров.

Обнаружено, что для образцов с узким ТЛ-пиком полуширина практически не претерпевает изменений при заполнении глубоких ловушек. Для образцов с широким пиком при температуре заполнения $1123 \mathrm{~K}$ наблюдается рост полуширины приблизительно на $10 \mathrm{~K}$ по сравнению с исходной величиной (погрешность измерения этого параметра не превышала $2 \mathrm{~K}$ ) (рис. 1). Этот рост продолжается до $T=1073 \mathrm{~K}$. Дальнейшее заполнение глубоких ловушек при температурах ниже $1073 \mathrm{~K}$ для образцов с широкими ТЛ-пиками приводило к уменьшению полуширины основного пика.

Кроме того, для образцов с широкими пиками экспериментально наблюдалось смещение температуры максимума ТЛ в область повышенных температур при за- полнении глубоких ловушек при $T=1123-973 \mathrm{~K}$. При дальнейшем уменьшении температуры облучения пик сдвигался в область более низких температур (рис. 2). У образцов с узкими пиками температура максимума ТЛ не претерпевала заметных изменений.

Разработка модельных представлений, описывающих экспериментальные результаты, проводилась на основе анализа кривых изменения ТЛ-чувствительности [11], полуширины (рис. 1) и температурного положения (рис. 2) пика при заполнении глубоких ловушек. Зонная схема модели ТЛ основного пика в исследуемых кристаллах представлена на рис. 3. В основу данной схемы положена модель, используемая в работе [11]. Как и ранее [11], основной пик ТЛ связан с опустошением электронной ловушки $N_{1}$ (узкий пик) или с одновременной ионизацией двух ловушек с близкими значениями энергии активации: низкотемпературной электронной $N_{1}$ и высокотемпературной дырочной $N_{2}$ (широкий пик). Уровень $M_{2}$ обусловлен присутствием очень глубокой ловушки, которая заполняется при облучении кристаллов УФ-излучением при $T>1000 \mathrm{~K}$ и имеет предположительно дырочную природу $[17,21]$. Уровень $M_{1}$ представляет глубокие электронные ловушки в исследуемых образцах, соответствующие ТЛ

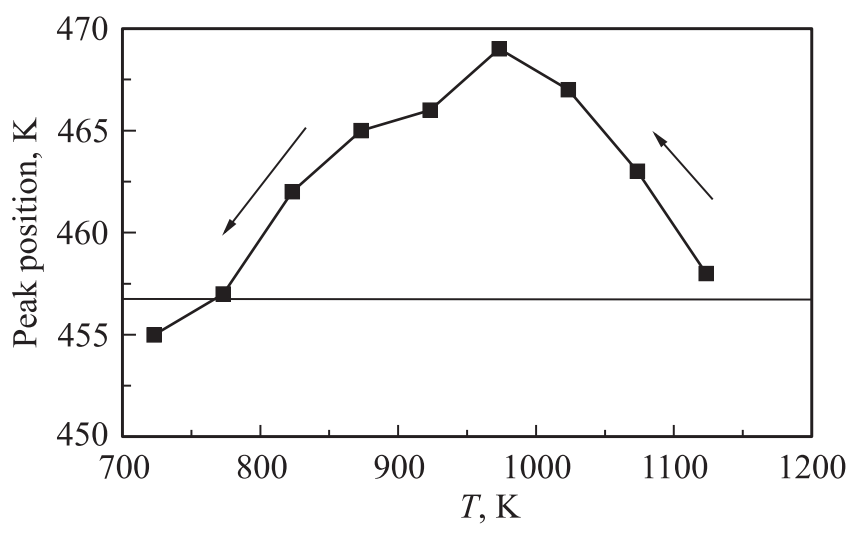

Рис. 2. Зависимость температуры максимума ТЛ от температуры облучения УФ-излучением для образцов с широким основным пиком. Горизонтальная линия соответствует исходному значению температуры пика.

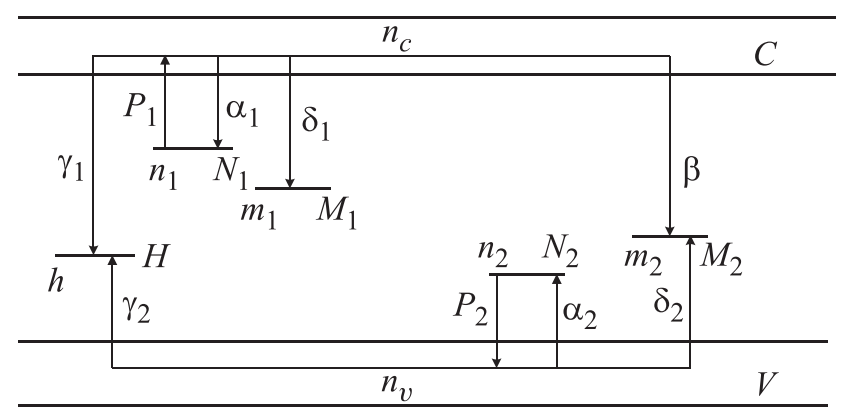

Рис. 3. Энергетическая зонная схема модели ТЛ основного пика, учитывающей дырочные ловушки. 
при $800-1000 \mathrm{~K}[18,19,21]$. Эти ловушки заполняются УФ-излучением при более низких температурах, чем дырочные [21]. В процессе облучения уровни $N_{1}, N_{2}$, $M_{1}, M_{2}$ заполняются носителями заряда разных знаков: электронами $\left(N_{1}, M_{1}\right)$ и дырками $\left(N_{2}, M_{2}\right)$. При термической стимуляции электроны и дырки, захваченные активными ловушками $N_{1}$ и $N_{2}$, инжектируются в зону проводимости $C$ и валентную зону $V$ соответственно. Релаксация возбужденных состояний как электронов, так и дырок может проходить по нескольким каналам: излучательная рекомбинация (переход $\gamma_{1}$ ), захват на глубокие ловушки (переходы $\delta$ ), повторный захват на ТЛ-активные ловушки (переходы $\alpha$ ), безызлучательная рекомбинация электронов с дырками на ловушке $M_{2}$ (переход $\beta$ ). Предполагается, что уровни $H, M_{1}$ и $M_{2}$ термически стабильны в температурном интервале регистрации ТЛ.

Хотя модель, используемая в работе [11], позволила описать особенности изменения ТЛ-чувствительности образцов с узкими и широкими ТЛ-пиками при заполнении глубоких ловушек, она не лишена некоторых недостатков. В ней ТЛ при $400-500 \mathrm{~K}$ обусловлена свечением электронного и дырочного центров люминесценции одновременно, что предполагает наличие в спектрах свечения ТЛ нескольких полос. Однако в реальных экспериментах, проведенных в настоящей работе (рис. 1 и 2) и ранее [11], все измерения ТЛ проводились только в одной полосе свечения $(410 \mathrm{~nm})$, связанной с $F$-центрами. Кроме того, согласно модельным представлениям [11], в данной полосе может наблюдаться только узкий пик ТЛ, обусловленный электронной ловушкой, что противоречит экспериментальным данным, поскольку уширенные пики ТЛ также наблюдаются в этой полосе свечения. В связи с этим потребовалось развитие модельных представлений, описывающих механизм ТЛ основного пика монокристаллов $\alpha-\mathrm{Al}_{2} \mathrm{O}_{3}$.

Особенностью усовершенствованной модели (рис. 3), отличающейся от предыдущей версии [11], является наличие только одного центра свечения, излучение которого обусловливает ТЛ как электронных, так и дырочных основных ловушек. В основу данных представлений была положена модель, предложенная в работах $[29,30]$ для описания сложной кривой термовысвечивания с двумя пиками ТЛ. В них ТЛ первого и второго пиков, обусловленных электронной и дырочной ловушками соответственно, возникала в результате релаксации только одного центра рекомбинации. По сравнению с моделью, описанной в работах $[29,30]$, предлагаемая в настоящей работе модель дополнительно учитывает процессы, связанные с глубокими центрами захвата. В качестве центра свечения $H$ в усовершенствованной модели выступает $F$-центр. В результате захвата дырки (переход $\gamma_{2}$ ) он превращается в $F^{+}$-центр. $F^{+}$-центр, захватив электрон (переход $\left.\gamma_{1}\right)$, трансформируется в возбужденный $F$-центр, который при переходе в основное состояние люминесцирует в полосе $410 \mathrm{~nm}$. Таким образом, в данной модели ТЛ дырочных ловушек может наблюдаться и в полосе свечения $F$-центров. Такой механизм ТЛ $F$-центров ранее не обсуждался в литературе и предложен в настоящей работе впервые.

Наша интерпретация результатов исследования влияния заполнения глубоких центров на параметры основного ТЛ-пика основана на известных механизмах конкурирующего взаимодействия ловушек, способных захватывать носители одного или разного знака (электроны и дырки) [22-24]. С ростом заполнения глубоких дырочных центров $M_{2}$ должен увеличиваться вклад дырочных ловушек $N_{2}$ в ТЛ основного пика за счет уменьшения вероятности перехода $\delta_{2}$ и роста вероятности перехода $\gamma_{2}$. Одновременно при заполнении глубоких ловушек $M_{2}$ уменьшается вклад в интенсивность ТЛ основного пика электронных ловушек $N_{1}$ за счет роста вероятности безызлучательного перехода $\beta$, который является конкурирующим процессом по отношению к излучательной рекомбинации $\gamma_{1}$. При заполнении глубоких электронных ловушек $M_{1}$ уменьшается вероятность перехода $\delta_{1}$, что приводит к росту числа актов излучательной рекомбинации (переход $\gamma_{1}$ ) и интенсивности ТЛ.

Учитывая приведенные рассуждения, можно констатировать, что при заполнении дырочных очень глубоких ловушек $M_{2}$ при температурах $1123-973 \mathrm{~K}$ наблюдаемое увеличение полуширины широких пиков ТЛ и смещение пика в высокотемпературную область связаны с ростом вклада в интенсивность ТЛ дырочных основных ловушек $N_{2}$. При этом интенсивность ТЛ меняется незначительно [11] за счет того, что одновременно происходит уменьшение вклада в ТЛ электронных ловушек $N_{1}$. Для образцов с узкими ТЛ-пиками, не содержащих в своем составе дырочных ловушек $N_{2}$ и характеризующихся отсутствием эффекта роста ТЛ-чувствительности при $T=1123-973 \mathrm{~K}$ [11], изменение формы и температурного положения пика не должно наблюдаться, что подтверждают наши эксперименты. Для образцов с узким ТЛ-пиком наблюдается падение ТЛ-чувствительности [11] за счет роста вероятности перехода $\beta$.

Дальнейшее заполнение электронных глубоких ловушек $M_{1}$ при температурах ниже $1000 \mathrm{~K}$ приводит к росту вклада в ТЛ основных электронных ловушек $N_{1}$ при практически неизменном вкладе дырочных $\left(N_{2}\right)$, что вызывает уменьшение полуширины пика и его сдвиг в низкотемпературную область в образцах с широкими пиками ТЛ. При этом наблюдается также рост ее интенсивности [11] в образцах с узкими и широкими ТЛ-пиками. Таким образом, модель (рис. 3) качественно описывает закономерности влияния заселенности глубоких ловушек на интенсивность, полуширину и положение максимума основного ТЛ-пика, подтверждая предположение о дырочной природе высокотемпературных основных ловушек.

Применимость модели (рис. 3) для количественного описания механизма ТЛ основного пика проверялась путем расчета зависимостей ТЛ-чувствительности от степени заполнения электронных и дырочных глубоких 
ловушек с последующим их сравнением с экспериментальными данными [11]. Математическое описание модели (рис. 3) определяется системой уравнений

$$
\begin{gathered}
d n_{1} / d t=-P_{1} n_{1}+\alpha_{1}\left(N_{1}-n_{1}\right) n_{c}, \\
d m_{1} / d t=\delta_{1}\left(M_{1}-m_{1}\right) n_{c}, \\
d n_{2} / d t=-P_{2} n_{2}+\alpha_{2}\left(N_{2}-n_{2}\right) n_{v}, \\
d h / d t=\gamma_{2}(H-h) n_{v}-\gamma_{1} h n_{c}, \\
d m_{2} / d t=-\beta m_{2} n_{c}+\delta_{2}\left(M_{2}-m_{2}\right) n_{v}, \\
d n_{c} / d t=-\gamma_{1} h n_{c}-\beta m_{2} n_{c}+P_{1} n_{1} \\
-\alpha_{1}\left(N_{1}-n_{1}\right) n_{c}-\delta_{1}\left(M_{1}-m_{1}\right) n_{c}, \\
d n_{v} / d t=-\gamma_{2}(H-h) n_{v}+P_{2} n_{2} \\
-\alpha_{2}\left(N_{2}-n_{2}\right) n_{v}-\delta_{2}\left(M_{2}-m_{2}\right) n_{v}, \\
\delta_{1}=\delta_{1}(T)=\delta_{0} \frac{c \exp (-W / k T)}{1+c \exp (-W / k T)} .
\end{gathered}
$$

В этих формулах $N_{1}, N_{2}, M_{1}, M_{2}\left(\mathrm{~cm}^{-3}\right)-$ максимально возможные концентрации зарядов в ТЛ-активных $(N)$ и глубоких ловушках $(M) ; n_{1}, n_{2}, m_{1}, m_{2}\left(\mathrm{~cm}^{-3}\right)-$ текущее заполнение уровней $N_{1}, N_{2}, M_{1}$ и $M_{2}$ coответственно; $\alpha_{1}, \delta_{1}, \gamma_{1}, \gamma_{2}, \alpha_{2}, \delta_{2}, \beta\left(\mathrm{cm}^{3} \cdot \mathrm{s}^{-1}\right)-$ коэффициенты переходов; $P_{1}, P_{2}$ - вероятности термической ионизации ТЛ-активных ловушек; $n_{c}\left(\mathrm{~cm}^{-3}\right)-$ концентрация свободных электронов в зоне проводимости $C ; n_{v}\left(\mathrm{~cm}^{3}\right)$ - концентрация свободных дырок в валентной зоне $V . P_{1}=S_{1} \exp \left(-E_{1} / k T\right)-$ вероятность выхода электрона в зону проводимости при термической ионизации электронных ТЛ-активных ловушек, $E_{1}-$ энергия активации процесса, $S_{1}$ - частотный фактор, $P_{2}=S_{2} \exp \left(-E_{2} / k T\right)$ - вероятность выхода дырок в валентную зону при термической ионизации дырочных ТЛ-активных ловушек, $E_{2}-$ энергия активации процесса, $S_{2}$ - частотный фактор. В модели, как и ранее [11], учитывается температурная зависимость вероятности захвата носителей на глубокие электронные ловушки $M_{1}$ (переход $\delta_{1}$, уравнение (8)). В (8) $W$ и $c$ - энергия активации и константа температурного тушения люминесценции [18,21]. $H-$ исходная концентрация $F$-центров, $h-$ концентрация $F^{+}$-центров. Множитель $(H-h)$ характеризует текущую концентрацию $F$-центров в произвольный момент времени.

Интенсивность ТЛ определяется выражением

$$
I(T)=\gamma_{1} h n_{c}
$$

Большинство параметров усовершенствованной модели идентично использованным ранее в работе [11] $\left(\alpha_{1}=\right.$ $=10^{-14} \mathrm{~cm}^{3} \cdot \mathrm{s}^{-1}, \alpha_{2}=10^{-14} \mathrm{~cm}^{3} \cdot \mathrm{s}^{-1}, \gamma_{1}=10^{-11} \mathrm{~cm}^{3} \cdot \mathrm{s}^{-1}$, $\gamma_{2}=10^{-11} \mathrm{~cm}^{3} \cdot \mathrm{s}^{-1}, \quad \delta_{0}=10^{-12} \mathrm{~cm}^{3} \cdot \mathrm{s}^{-1}, \quad \delta_{2}=$ $=10^{-12} \mathrm{~cm}^{3} \cdot \mathrm{s}^{-1}, \beta=3 \cdot 10^{-12} \mathrm{~cm}^{3} \cdot \mathrm{s}^{-1}, c=10^{11}, W=$ $=1.1 \mathrm{eV}, M_{1}=10^{14} \mathrm{~cm}^{-3}, M_{2}=10^{14} \mathrm{~cm}^{-3}, N_{1}=10^{13} \mathrm{~cm}^{-3}$, $N_{2}=10^{13} \mathrm{~cm}^{-3}, E_{1}=1.30 \mathrm{eV}, E_{2}=1.35 \mathrm{eV}, S_{1}=10^{13} \mathrm{~s}^{-1}$, $S_{2}=10^{13} \mathrm{~s}^{-1}$ ). Уменьшены на порядок величины $\gamma_{2}$ и $\delta_{2}$,

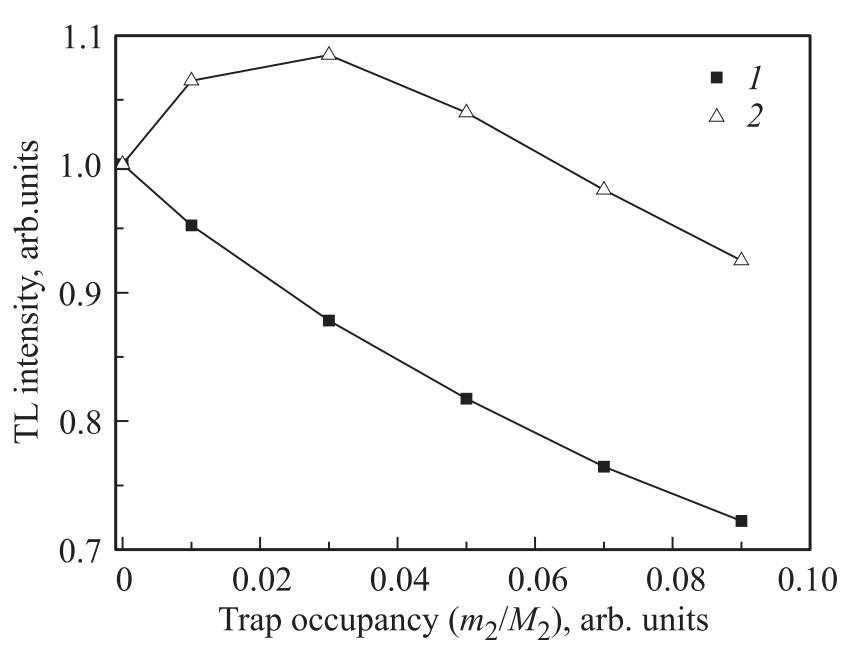

Рис. 4. Зависимости интенсивности ТЛ от степени заполнения глубоких дырочных ловушек для образцов с узким (1) и широким (2) пиками.

характеризующие дырочные процессы, чтобы обеспечить качественное выполнение наблюдаемых закономерностей влияния глубоких ловушек на параметры ТЛ основного пика. Также претерпели незначительные изменения параметры $S_{2}, E_{1}$ и $E_{2}$ для улучшения совпадения экспериментальных и теоретических кривых ТЛ. Выбраны значения $S_{2}=3 \cdot 10^{13} \mathrm{~s}^{-1}, E_{1}=$ $=1.25 \mathrm{eV}, E_{2}=1.32 \mathrm{eV}$. Начальные концентрации центров свечения задавались исходя из экспериментальных данных измерения оптического поглощения исследуемых кристаллов [2,27]. При этом концентрация $F$-центров составила величину $H=10^{17} \mathrm{~cm}^{-3}$, а $F^{+}$-центров - $h_{0}=10^{14} \mathrm{~cm}^{-3}$.

В рамках предложенного усовершенствованного варианта модели рассчитаны кривые ТЛ при пустых глубоких ловушках для двух случаев. В первом основной пик был обусловлен моноэнергетической электронной ловушкой (узкий пик). Во втором он представлял собой суперпозицию электронной и дырочной ловушек (широкий пик). Результаты расчета показали, что полуширина основного ТЛ-пика составила величину $32 \mathrm{~K}$ в первом случае и $48 \mathrm{~K}$ во втором, что очень близко к экспериментальным диапазонам полуширин для узких и широких ТЛ-пиков в исследуемых образцах. При этом расчетные значения температур максимумов пиков для обоих случаев (449 и $457 \mathrm{~K}$ соответственно) оказались близки к величинам, наблюдаемым экспериментально.

Расчетные зависимости ТЛ-чувствительности кристаллов к излучению от степени заполнения дырочных глубоких ловушек $M_{2}$ и электронных глубоких ловушек $M_{1}$ приведены на рис. 4 и 5. Расчет проводился для узкого и широкого ТЛ-пиков. Результаты показывают, что при заполнении дырочных глубоких ловушек (рис. 4) чувствительность кристаллов существенно уменьшается только для образцов с узким пиком, что и наблюдалось ранее экспериментально [11]. Незначительный рост ТЛ 


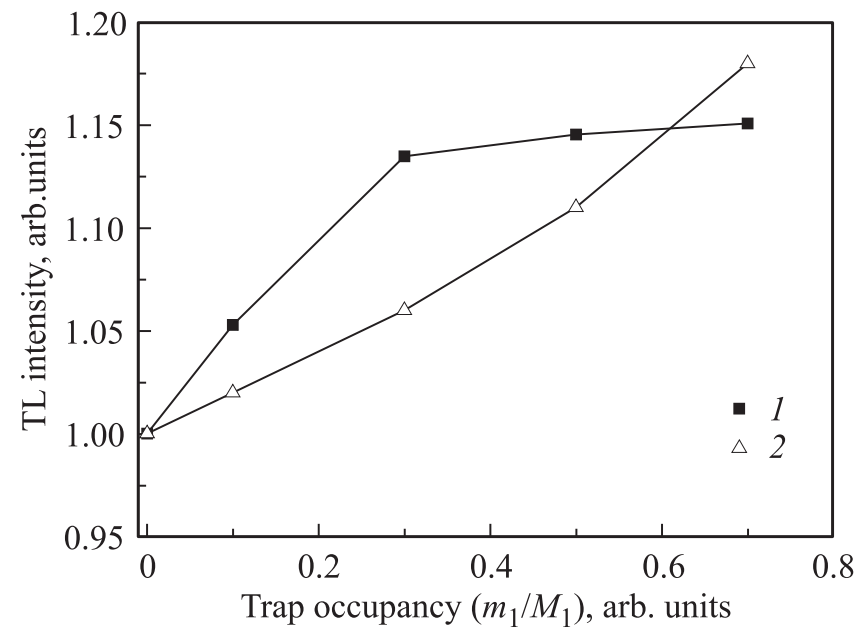

Рис. 5. Зависимости интенсивности ТЛ от степени заполнения глубоких электронных ловушек для образцов с узким (1) и широким (2) пиками.

образцов с широкими пиками при малой заселенности глубоких ловушек $M_{2}$ (начальный участок кривой 2) может быть интерпретирован следующим образом. Рост интенсивности ТЛ основного пика, обусловленный заполнением дырочных глубоких ловушек $M_{2}$, возникает за счет перехода $\delta_{2}$, вероятность которого пропорциональна произведению коэффициента перехода на концентрацию пустых ловушек $\left(M_{2}-m_{2}\right)$. В свою очередь падение выхода ТЛ определяется переходом $\beta$, вероятность которого равна произведению коэффициента перехода на концентрацию заполненных ловушек $\left(m_{2}\right)$. С учетом близости в пределах порядка значений $\delta_{2}$ и $\beta$ при малой заселенности ловушек $M_{2}$ вероятность перехода $\beta$ оказывается много меньше вероятности перехода $\delta_{2}$, что обусловливает рост ТЛ-чувствительности. При дальнейшем заполнении ловушек $M_{2}$ растет вероятность перехода $\beta$, что приводит к появлению спадающего участка на кривой 2 изменения ТЛ-чувствительности (рис. 4).

Расчеты также показали, что заполнение глубоких электронных ловушек $M_{1}$ приводит к росту ТЛ-чувствительности для всех исследуемых образцов (рис. 5), что также согласуется с экспериментальными данными [11].

Таким образом, усовершенствованная модель, как и предыдущая ее версия [11], позволяет количественно описать различный характер изменения ТЛ-чувствительности образцов с узким и широким основным пиком при вариации степени заполнения глубоких ловушек. Вместе с тем предложенная в настоящей работе усовершенствованная модель является более корректной, поскольку она объясняет механизм ТЛ процесса с учетом ее спектральных особенностей.

Изложенные выше результаты были посвящены обоснованию дырочной природы ловушек, вызывающих уширение ТЛ-пика при 400-500 K в анион-дефектном оксиде алюминия, с помощью анализа конкурирующих процессов с участием глубоких ловушек. Вместе с тем известно, что полуширина основного пика оказывает влияние и на ТЛ мелких ловушек, обусловливающих пик ТЛ при $350 \mathrm{~K}$ в исследуемых кристаллах [25,26]. В работе [25] обнаружено, что существует корреляция между интенсивностью ТЛ-пика при $350 \mathrm{~K}$, обусловленного мелкими ловушками, и полушириной основного пика при 400-500 К. Для образцов с малой полушириной основного пика $(35-45 \mathrm{~K})$ величина пика при $350 \mathrm{~K}$ могла изменяться в больших пределах в зависимости от концентрации мелких ловушек. Вместе с тем в образцах с широким основным пиком интенсивность пика при $350 \mathrm{~K}$ очень мала для всех образцов. Эта закономерность наблюдалась многократно для образцов с различной ТЛ-чувствительностью в основном пике, однако не была интерпретирована. Объяснение данного эффекта может быть дано с учетом предположения о дырочной природе высокотемпературной части ловушек, обусловливающих основной ТЛ-пик.

Энергетическая зонная схема, описывающая ТЛ мелких ловушек, приведена на рис. 6. Здесь $N_{3}-$ мелкая электронная ловушка, ответственная за пик ТЛ при $350 \mathrm{~K}$, которая имеет электронную природу [25], $H$ центр свечения. Как и ранее в модели, приведенной на рис. 3, предполагается, что основной пик в общем случае обусловлен суперпозицией двух ловушек: электронной $N_{1}$ и дырочной $N_{2}$. Переход $P_{3}$ описывает термическую ионизацию мелких ловушек при нагреве образца, $\alpha_{3}$ - повторный захват на мелкие ловушки, $\alpha_{1}$ - захват на электронные ловушки $N_{1}, \gamma-$ излучательная рекомбинация. Термическим освобождением носителей из основных ловушек в диапазоне температур высвечивания ТЛ-пика при $350 \mathrm{~K}$ будем пренебрегать.

Можно предположить, что в процессе термического опустошения мелких ловушек часть электронов безызлучательно рекомбинирует с дырками на ловушках высокотемпературной части основного ТЛ-пика (переход $\beta$ ). Этот безызлучательный переход является процессом, конкурирующим с рекомбинацией носителей на цен-

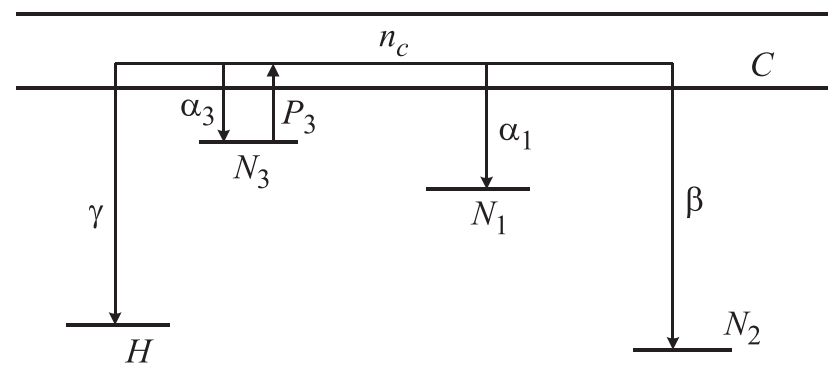

V

Рис. 6. Энергетическая зонная схема модели конкурирующего взаимодействия мелких и основных ловушек. 


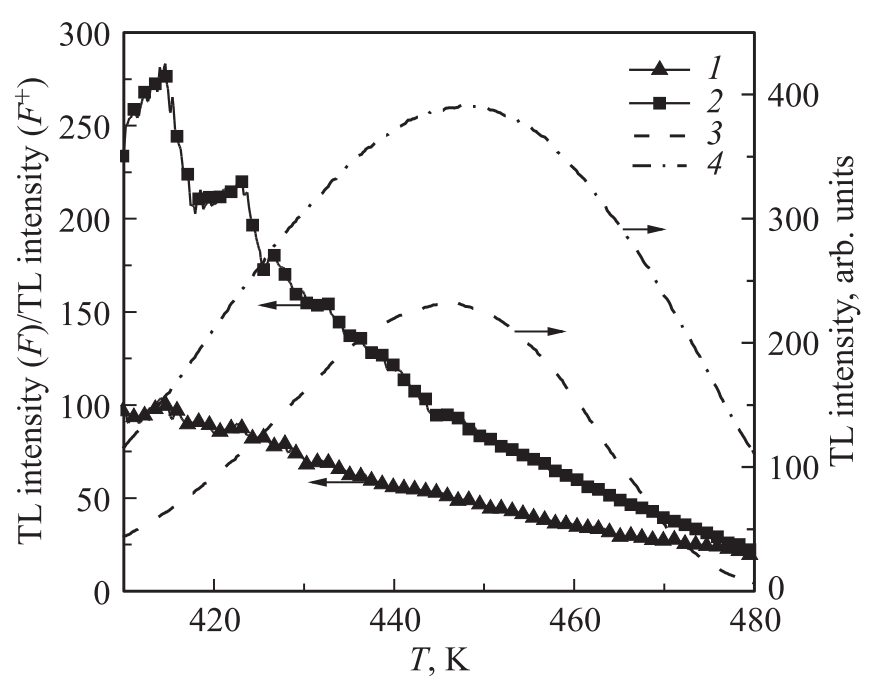

Рис. 7. Зависимость отношения интенсивностей ТЛ в полоcax свечения $F$ - и $F^{+}$-центров от температуры в пределах основного пика для образцов с узкими (1) и широкими (2) пиками. 3 и $4-$ кривые ТЛ образцов с узким и широким пиком соответственно.

трах свечения (переход $\gamma$ ), и приводит к уменьшению интенсивности ТЛ-пика при $350 \mathrm{~K}$, что и наблюдалось экспериментально для образцов с широким основным пиком [25]. Таким образом, введение в рассмотрение дырочных ловушек высокотемпературной части основного ТЛ-пика позволяет интерпретировать и особенности ТЛ мелких центров захвата. Следует отметить, что модель (рис. 6) в рамках представлений о конкурирующих электронных ловушках объясняет также ранее изученные эффекты изменения выхода ТЛ-пика при $350 \mathrm{~K}$ после заполнения/опустошения основных электронных ловушек [31].

В пользу дырочной природы ловушек, обусловливающих высокотемпературную часть основного пика, свидетельствуют и результаты других экспериментов, напрямую не связанных с исследованиями конкурирующих процессов взаимодействия ловушек. Измерялась ТЛ основного пика в полосах свечения $F$ - $(410 \mathrm{~nm})$ и $F^{+}$-центров $(330 \mathrm{~nm})$. На рис. 7 приведены зависимости отношения интенсивностей ТЛ в указанных полосах от температуры, изменяемой в диапазоне высвечивания основного пика, для образцов с узким (кривая 1) и широким (кривая 2) пиками. Видно, что в пределах пика наблюдается падение величины отношения интенсивностей ТЛ $F$ - и $F^{+}$-центров. Эта закономерность ранее уже наблюдалась в анион-дефектных кристаллах оксида алюминия, легированных магнием [15] и одновременно магнием и хромом [32]. Новым важным результатом настоящей работы является обнаружение этого эффекта в номинально чистых дозиметрических кристаллах $\alpha-\mathrm{Al}_{2} \mathrm{O}_{3}$, а также зависимости степени его проявления от формы основного ТЛ-пика. Из рис. 7 видно, что величина падения отношения интенсивностей полос ТЛ
$F$ - и $F^{+}$-центров больше для образцов с широкими пиками, чем для образцов с узкими.

Полученные результаты можно интерпретировать следующим образом. Уменьшение отношения интенсивностей ТЛ $F$ - и $F^{+}$-центров в пределах пика при 400-500 K свидетельствует о росте интенсивности свечения $F^{+}$-центров в его спадающей высокотемпературной части. Данный факт может указывать на то, что ТЛ этой части пика обусловлена в основном дырочными ловушками. При этом ТЛ возникает согласно реакции $F+h^{+}=F^{+*}=F^{+}+h v(330 \mathrm{~nm})$.

Поскольку в образцах с широкими ТЛ-пиками интенсивность свечения $F^{+}$-центров изменяется сильнее, чем в случае $F$-центров, можно ожидать, что и вклад дырочных ловушек в ТЛ-пика при 400-500 K (особенно в высокотемпературную его часть) будет наибольшим в образцах с уширенными ТЛ-пиками. При этом нельзя исключить наличие среди ловушек, ответственных за высокотемпературную часть основного ТЛ-пика, и электронных компонент с малой концентрацией, поскольку в данном температурном диапазоне наблюдается свечение не только $F^{+}$-, но и $F$-центров.

Установление взаимосвязи дырочных ловушек основного ТЛ-пика с собственными либо примесными дефектами кристаллической решетки требует дальнейших исследований. В работе [4] показано, что уширение ТЛ-пика не связано с дефектами деформационного происхождения, а может быть обусловлено присутствием в исследуемых образцах следов неконтролируемых примесей. Вариация содержания примесей, обусловленная различными условиями выращивания монокристаллов, приводит к изменению концентрации примесных ловушек. Такие ловушки могут давать сигнал ТЛ вблизи температурного диапазона высвечивания основного пика, искажая его форму и вызывая, в частности, рост его полуширины. В работе [7] в качестве такой примеси упоминались ионы магния. Получен также ряд результатов, показывающих возможную роль в формировании дырочных ловушек примеси титана. В частности, обнаружено, что вблизи температурного диапазона высвечивания основного пика регистрируется пик ТЛ при $480 \mathrm{~K}$ после импульсного электронного облучения номинально чистых монокристаллов $\alpha-\mathrm{Al}_{2} \mathrm{O}_{3}$ и кристаллов оксида алюминия, легированных титаном [33]. При этом величина максимума ТЛ данного пика коррелировала с интенсивностью полосы импульсной катодолюминесценции при $650-750 \mathrm{~nm}$, обусловленной ионами $\mathrm{Ti}^{3+}$. Существуют также данные о прямой взаимосвязи интенсивности этой полосы и полуширины основного пика ТЛ [11]. Другой причиной уширения энергетического спектра ловушек, ответственных за пик ТЛ при 400-500 К в исследуемых кристаллах, кроме ионов $\mathrm{Ti}^{3+}$ могут быть ионы кремния. Как и в случае с титаном, ионы кремния могут обусловливать дополнительный ТЛ-пик, близкий по температурному положению к основному максимуму. Такой пик при $475 \mathrm{~K}$ (скорость нагрева $10 \mathrm{~K} / \mathrm{s}$ ) был обнаружен в кристаллах оксида алюминия, содержащих повышенные 
концентрации примеси кремния $(0.2 \%)$ после облучения электронным пучком (100 Gy) [34]. В работе [35] пик ТЛ при $191^{\circ} \mathrm{C}$ (скорость нагрева $4 \mathrm{~K} / \mathrm{s}$ ), близкий по температурному положению к основному ТЛ-пику, был обнаружен в образцах $\mathrm{Al}_{2} \mathrm{O}_{3}: \mathrm{Si}, \mathrm{Ti}$. Кроме того, было обнаружено, что введение кремния в кристаллическую решетку оксида алюминия приводило к появлению сложной структуры основного ТЛ-пика при $400-500 \mathrm{~K}$ и его уширению в низкотемпературную область [36].

\section{4. Заключение}

Получены новые экспериментальные доказательства дырочной природы ловушек, ответственных за уширение основного ТЛ-пика при $400-500 \mathrm{~K}$ в анион-дефектных монокристаллах оксида алюминия в высокотемпературную область. Присутствие данных ловушек доказывается на основе анализа экспериментальных зависимостей параметров ТЛ основного пика (интенсивности, температурного положения и полуширины) от степени заполнения глубоких ловушек в образцах с узкими и широкими ТЛ-пиками. Дана теоретическая интерпретация этих зависимостей в рамках кинетической модели, учитывающей электронные и дырочные процессы конкуренции в захвате носителей заряда между основными и глубокими ловушками. Представление о дырочных высокотемпературных основных ловушках позволяет также интерпретировать взаимосвязь между интенсивностью ТЛ мелких ловушек при $350 \mathrm{~K}$ и полушириной основного пика в рамках модели конкурирующих центров. Дырочная природа высокотемпературных основных ловушек подтверждена также при анализе спектральных особенностей ТЛ образцов с различной полушириной пика при $400-500 \mathrm{~K}$.

\section{Список литературы}

[1] М.С. Аксельрод, В.С. Кортов, И.И. Мильман, Е.А. Горелова, А.А. Борисов, Л.М. Затуловский, Д.Я. Кравецкий, И.Е. Березина, Н.К. Лебедев. Изв. АН СССР. Сер. физ. 52, 1981 (1988).

[2] S.W.S. McKeever, M.S. Akselrod, L.E. Colyott, N. Agersnap Larsen, J.C. Polf, V. Whitley. Radiat. Prot. Dosim. 84, 163 (1999).

[3] K.H. Lee, J.H. Crawford. Phys. Rev. B 15, 4065 (1977).

[4] В.С. Кортов, И.И. Мильман, С.В. Никифоров. ФТТ 39, 1538 (1997).

[5] A.E. Akselrod, M.S. Akselrod. Radiat. Prot. Dosim. 100, 217 (2002).

[6] G.I. Dallas, D. Afouxenidis, E.C. Stefanaki, N.F. Tsagas, G.S. Polymeris, N.C. Tsirliganis, G. Kitis. Phys. Status Solidi A 205, 1672 (2008).

[7] G.S. Polymeris, G. Kitis. Appl. Radiat. Isotopes 70, 2478 (2012).

[8] L.E. Colyott, M.S. Akselrod, S.W.S. McKeever. Radiat. Prot. Dosim. 65, 263 (1996).

[9] F.D. Walker, L.E. Colyott, N. Agersnap Larsen, S.W.S. McKeever. Radiat. Meas. 26, 711 (1996).
[10] E.G. Yukihara, V.H. Whitley, J.C. Polf, D.M. Klein, S.W.S. McKeever, A.E. Akselrod, M.S. Akselrod. Radiat. Meas. 37, 627 (2003).

[11] С.В. Никифоров, В.С. Кортов, А.А. Носаль, Е.В. Моисейкин. ФТТ 53, 2032 (2011).

[12] V.H. Whitley, S.W.S. McKeever. Radiat. Prot. Dosim. 100, 61 (2002).

[13] E.G. Yukihara, V.H. Whitley, S.W.S. McKeever, A.E. Akselrod, M.S. Akselrod. Radiat. Meas. 38, 317 (2004).

[14] G.I. Dallas, G.S. Polymeris, E.C. Stefanaki, D. Afouxenidis, N.C. Tsirliganis, G. Kitis. Radiat. Meas. 43, 335 (2008).

[15] B.J. Jeffries, J.D. Brewer, G.P. Summers. Phys. Rev. B 24, 6074 (1981).

[16] M.S. Akselrod, E.A. Gorelova. Nucl. Tracks Radiat. Meas. 21, 143 (1993).

[17] S.V. Nikiforov, V.S. Kortov, S.V. Zvonarev, E.V. Moiseykin, M.G. Kazantseva. Radiat. Meas. 71, 74 (2014).

[18] И.И. Мильман, В.С. Кортов, С.В. Никифоров. ФТТ 40, 229 (1998).

[19] И.И. Мильман, Е.В. Моисейкин, С.В. Никифоров, С.В. Соловьев, И.Г. Ревков, Е.Н. Литовченко. ФТТ 50, 1991 (2008).

[20] A.I. Surdo, R.M. Abashev, I.I. Milman, E.V. Moiseykin. Radiat. Meas. 90, 192 (2016).

[21] S.V. Nikiforov, V.S. Kortov. Radiat. Meas. 45, 527 (2010).

[22] R. Chen, G. Fogel. Radiat. Prot. Dosim. 47, 23 (1993).

[23] R. Chen, G. Fogel, C.K. Lee. Radiat. Prot. Dosim. 65, 63 (1996).

[24] V. Pagonis, R. Chen, J.L. Lawless. Radiat. Meas. 42, 198 (2007).

[25] V.S. Kortov, S.V. Nikiforov, E.Z. Sadykova. Funct. Mater. 2, 282 (2005).

[26] D.R. Mishra, M.S. Kulkarni, K.P. Muthe, C. Thinaharan, M. Roy, S.K. Kulshreshtha, S. Kannan, B.C. Bhatt, S.K. Gupta, D.N. Sharma. Radiat. Meas. 42, 170 (2007).

[27] M.S. Akselrod, V.S. Kortov, E.A. Gorelova. Radiat. Prot. Dosim. 47, 159 (1993).

[28] A.S. Vokhmintsev, M.G. Minin, A.M.A. Henaish, I.A. Weinstein. Measurement 66, 90 (2015).

[29] R. Chen, V. Pagonis, J.L. Lawless. Radiat. Meas. 43, 162 (2008).

[30] J.L. Lawless, R. Chen, V. Pagonis. J. Phys. D 42, 155409 (2009).

[31] В.С. Кортов, С.В. Никифоров, Э.З. Садыкова. Изв. вузов. Физика 2, 89 (2006).

[32] M.S. Akselrod, V.S. Kortov. Radiat. Prot. Dosim. 33, 123 (1990).

[33] I.I. Milman, E.V. Moiseykin, S.V. Nikiforov, S.G. Mikhailov, V.I. Solomonov. Radiat. Meas. 38, 443 (2004).

[34] A.C. Lucas, B.K. Lucas. Radiat. Prot. Dosim. 85, 455 (1999).

[35] B.C. Bhatt, P.S. Page, N.S. Rawat, B.S. Dhabekar, D.R. Mishra, M.S. Kulkarni. Radiat. Meas. 43, 327 (2008).

[36] Т.С. Бессонова, Т.И. Гимадова, И.А. Тале, Л.А. Аввакумова, Л.А. Литвинов. ЖПС 54, 433 (1991). 